\title{
Surface instability of multipulse laser ablation on a metallic target
}

\author{
L. K. Ang, Y. Y. Lau, ${ }^{\text {a) }}$ R. M. Gilgenbach, ${ }^{\text {a) }}$ H. L. Spindler, ${ }^{\text {b) }}$ J. S. Lash, ${ }^{\text {c) }}$ \\ and S. D. Kovaleski \\ Intense Energy Beam Interaction Laboratory, Department of Nuclear Engineering \\ and Radiological Sciences, The University of Michigan, Ann Arbor, Michigan 48109-2104
}

(Received 28 April 1997; accepted for publication 6 January 1998)

\begin{abstract}
Large scale wavelike patterns are observed on an aluminum surface after it is ablated by a series of $\mathrm{KrF}$ laser pulses $\left(248 \mathrm{~nm}, 40 \mathrm{~ns}, 5 \mathrm{~J} / \mathrm{cm}^{2}\right)$. These surface structures have a wavelength on the order of $30 \mu \mathrm{m}$, much longer than the laser wavelength. We postulate that these wave patterns are caused by the Kelvin-Helmholtz instability at the interface between the molten aluminum and the plasma plume. A parametric study is given in terms of the molten layer's thickness and of the spatial extent and kinetic energy density in the laser-produced plasma plume. Also included is an estimate of the cumulative growth in a multipulse laser ablation experiment. These estimates indicate that the Kelvin-Helmholtz instability is a viable mechanism for the formation of the large scale structures. (C) 1998 American Institute of Physics. [S0021-8979(98)00408-3]
\end{abstract}

\section{INTRODUCTION}

Pulsed-laser deposition (PLD) is an efficient and versatile thin film deposition technique. ${ }^{1}$ The major concern of PLD is the particulate formation that leads to the splashing of materials on the target. There is considerable evidence that large particulate formation is preceded by the formation of wavelike structures on the ablated surface. ${ }^{2}$ The surface modification by cumulative laser irradiation also affects both the film composition and deposition rate. ${ }^{3}$ The mechanisms of the laser sputtering of materials have been studied. ${ }^{4}$

Roughly speaking, there are two types of surface structures resulting from laser irradiation. The first type is of small scale, on the order of the laser wavelength. They are observed during the first few pulses or when the laser fluence is below the damage threshold of the surface. In general, they are more dependent on the surface condition, such as the optical and thermophysical properties of the material. There are existing theories for the formation of these small scale periodic surface structures. ${ }^{5}$ The proposed mechanisms include inhomogeneous energy deposition due to the interference of the incident laser beam and the surface scattering field. Excitation of surface plasmons can produce anomalous absorption under high fluence irradiation, ${ }^{6}$ and the laserdriven corrugation instability leads to a strong coupling when the critical depth reaches the pump laser wavelength.

The second type of surface structures, the object of the present study, has a scale much greater than the pump laser wavelength. These large surface structures ${ }^{7,8}$ appear when the laser fluence is above the damage threshold and the target is heated beyond the vapor phase to the plasma state. In the multipulse experiments, ${ }^{2,9-16}$ a $\mathrm{KrF}$ laser $(248 \mathrm{~nm}, 40 \mathrm{~ns}$, $5-10 \mathrm{~J} / \mathrm{cm}^{2}$ ) is incident on an aluminum target. From the scanning electron microscope (SEM) pictures of the dam-

\footnotetext{
a) Also at: Applied Physics Program, University of Michigan, Ann Arbor, MI 48109.

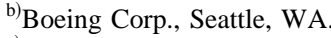

${ }^{c}$ Sandia National Laboratory, Albuquerque, NM.
}

aged target surface for a number of pulses up to 4200 shots, we observe a relatively uniform ripple formation in the center of the laser spot, with large surface structures formed $(50-100 \mu \mathrm{m})$ in the outer edge. The spacing of the ripple is much larger than the laser wavelength, on the order of 30 $\mu \mathrm{m}$ (see Figs. 1 and 2 below).

The laser-produced plasma plumes have several effects on laser-target interactions. They might strengthen the large scale structures on the one hand (see below), and they might absorb or reflect the laser energy, ${ }^{17}$ thereby preventing the laser light from reaching the surface, with the result of reducing the thickness of the molten layer. Contrary to the case of small scale surface structures, the surface conditions of the pre-ablated metal, as well as whatever interference effects with the incident laser light, are probably less crucial in the formation of the large scale structures, which were not observed for polymers and other materials using this $\mathrm{KrF}$ laser.

The presence of large scale surface structures must have been created by a rather significant energy reservoir. The fairly regular wavelike patterns look much like the winddriven surface wave in a pond (see Fig. 1). This recognition naturally directs our attention to the Kelvin-Helmholtz instability ${ }^{18}$ as a candidate for the creation of similar wavelike structures. In our case, the molten aluminum plays the role of "water," and the "wind" comes from expansion of the high pressure plasma plume. The large surface structures occur when the surface waves (coming from the center of the laser spot) are splashed and solidified at the edge. We may tentatively identify the Kelvin-Helmholtz instability as a viable candidate if the computed growth rate may explain the buildup of the large scale structures during the multipulse ablation, upon using a reasonable set of parameters consistent with the experiments.

In addition to Kelvin-Helmholtz $(\mathrm{KH})$ instability, we have also studied the possibilities of other hydrodynamic instabilities, such as capillary wave (CW) and RayleighTaylor (RT) instability. Brailovsky et al. ${ }^{8}$ considered all three instabilities for the mechanisms of melt droplets. For 


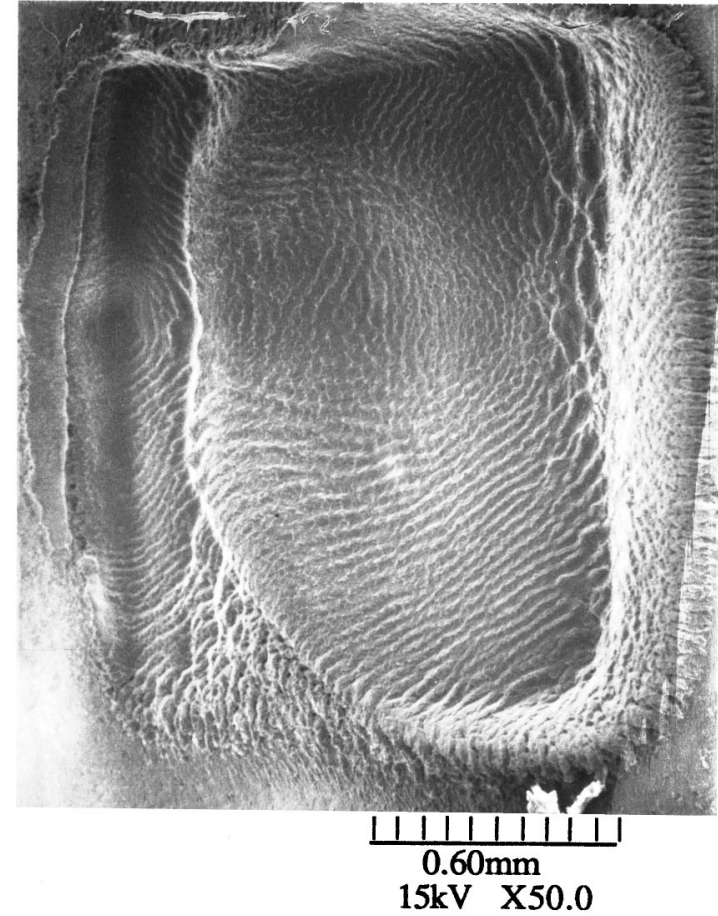

FIG. 1. A SEM picture (at $50 \times$ magnification) of the laser ablation spot of $1000 \mathrm{KrF}$ excimer laser pulses on an aluminum target at a fluence of $10 \mathrm{~J} / \mathrm{cm}^{2}$ [from Ref. 10].

CW instability, they considered a subsonic vapor evaporated from the surface with a perpendicular expansion velocity of order $10^{4} \mathrm{~cm} / \mathrm{s}$, which is two orders of magnitude lower than our measured velocity (on the order of $\mathrm{cm} / \mu \mathrm{s}$ ) in the experiments $^{10-16}$ (see below). Their calculation for the case of bismuth ablated by laser intensity of $10^{9} \mathrm{~W} / \mathrm{cm}^{2}$ yielded a maximum instability increment $\left(\lambda_{m}\right)$ of $0.1 \mu \mathrm{m}$, which is much lower than our experimental value of about $30 \mu \mathrm{m}$.

Unlike the RT instability discussed by Brailovsky, ${ }^{19}$ conventional RT instability model has been used to calculate

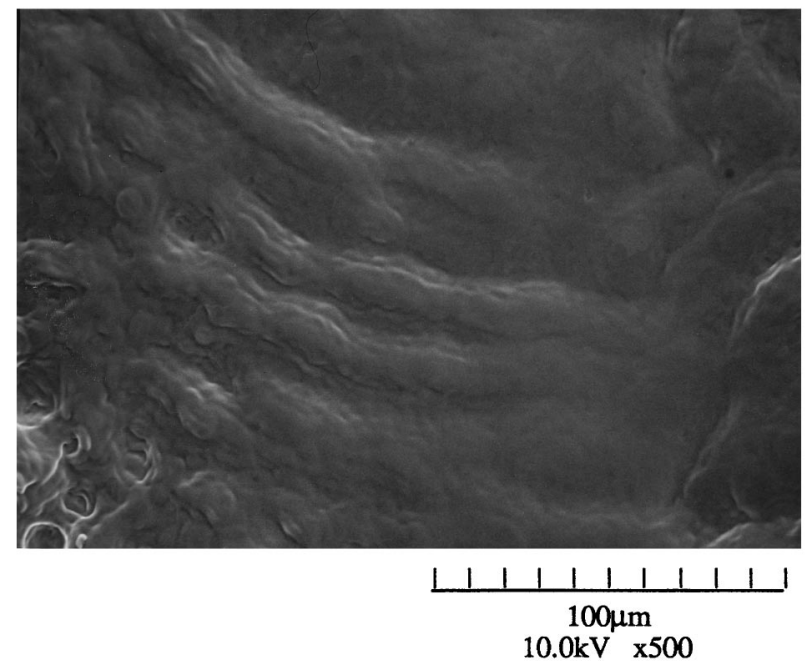

FIG. 2. A SEM picture (at $500 \times$ magnification) in the middle of a damaged Al target irradiated by 250 pulses of $\mathrm{KrF}$ excimer laser $(248 \mathrm{~nm}, 40 \mathrm{~ns}$, $5 \mathrm{~J} / \mathrm{cm}^{2}$ on a pure aluminum target in vacuum condition $(<2$ $\times 10^{-5}$ Torr) . $\lambda_{m}$; it equals $4 \mu \mathrm{m}$ by estimating an acceleration force $(\alpha)$ of $3.2 \times 10^{9} \mathrm{~m} / \mathrm{s}^{2}$ (Ref. 20) in the liquid-vapor interface. Moreover, the RT instability usually does not have its signature in the form of a very regular wave pattern as exhibited in Fig. 1, although this instability might well be a candidate for the formation of the individual droplets of molten materials at the edge of the laser spot, ${ }^{2}$ but droplet formation is beyond the scope of the present paper.

The growth rate of the thermocapillary instability ${ }^{21}$ is too slow to initiate the large scale structures. The corrugation instability of plane evaporation waves ${ }^{21}$ in liquid also grows too slowly. The above considerations of small $\lambda_{m}$ (less than $10 \mu \mathrm{m})$ and slow growth rate help us to eliminate the other macroscopic instabilities in favor of $\mathrm{KH}$ instability. We note that our model is different from the one previously considered by Brailovsky et $a .^{8}$ in that their $\mathrm{KH}$ instability mechanism is caused by the perpendicular plume velocity streaming along the vertical column of the molten layer. More specifically, Brailovsky examined the $\mathrm{KH}$ instability on the vertical columns that are already formed. Here we analyze how these vertical columns are formed in the first place: the parallel wind leads to growth of the vertical columns via the $\mathrm{KH}$ instability. Once these vertical columns are formed, the (vertical) wind then provides secondary ripples on these columns. Brailovky analyzed the secondary ripples thus formed. It is clear that when the amplitude of the vertical columns is small (which is the case during their initial growth that is analyzed here), Brailovsky's mechanism is inoperative.

The uncertainties in the parameters, such as the depth of the molten layer and the density and velocity profiles of the plasma wind at the liquid-plasma interface, force us to use a semiemperical approach on a much simplified model. We use a one-dimensional model with conveniently chosen density and velocity profiles for the two media: molten liquid and plasma wind. We postulate that the wavelike pattern that is observed in the experiment corresponds to the wave number that yields the highest growth rate. We then examine the dependence of this maximum growth on the parameters. We next evaluate the cumulative growth for a sequence of laser pulses, assuming that between pulses, the wave pattern solidifies quickly so that the frozen pattern serves as the initial condition at the time the next laser pulse arrives. The ranges of the chosen parameters are guided by extensive, previous experimental measurements of plasma plume parameters. ${ }^{10-16}$ From Ref. 12 (Figs. 9i, j), one can infer a transverse plasma wind velocity of $0.18 \mathrm{~cm} / \mu \mathrm{s}$.

Finally, we remark that the laser inhomogeneity is unlikely to produce wavelike patterns so regular over a broad front (cf. Fig. 1) and these have not been seen in our previous experiments on polymer and ceramic ablation.

\section{EXPERIMENTAL CONFIGURATION AND DATA}

The laser ablation plume was produced by focusing a pulse from a $\mathrm{KrF}$ excimer laser $(248 \mathrm{~nm}, 40 \mathrm{~ns},<1.2 \mathrm{~J})$ on aluminum targets. The analyzed samples consisted of seven 99.999\% pure solid aluminum targets. The targets were placed at a ninety degree angle to the incident laser beam. The laser focal spot was 2.0 by $2.5 \mathrm{~mm}$, or $0.05 \mathrm{~cm}^{2}$ in area. 
The fluence was measured at regular intervals by inserting a calorimeter in front of the $\mathrm{KrF}$ laser beam. The average laser fluence at the target was approximately $5 \mathrm{~J} / \mathrm{cm}^{2}$.

For these experiments, the stainless-steel vacuum system was evacuated using a rotary pump-backed turbomolecular pump. The pressure remained less than $2 \times 10^{-5}$ Torr during the laser ablation of aluminum.

Progressive levels of damage, ranging from 5 up to 4200 excimer laser pulses, were produced on each of seven targets. The resulting damage on the targets was investigated by placing each target in an SEM. At each stage of damage, an SEM image was taken of the entire laser focal spot and a second SEM image was taken at a higher magnification in the middle of the damaged area. From our results, the significant uniform ripple began to occur at 250 laser pulses irradiation. Here, we only present the SEM image $(500 \times)$ in the middle of the damaged area at 250 laser pulses (see Fig. 2 ). Figure 1 shows the damage in the middle of the spot at 1000 pulses and $50 \times$ magnification.

This $\mathrm{KrF}$ laser ablated $\mathrm{Al}$ plasma has been extensively characterized in our previous research. ${ }^{11-16}$ Many different diagnostics have been used to investigate the expansion velocity of the laser ablation plumes, such as resonant holographic interferometry, ${ }^{11,12}$ resonance absorption photography, ${ }^{11,13,14}$ schlieren photography, ${ }^{14,15}$ and laser deflection. ${ }^{11,16}$ The electron density has been measured by laser deflection. ${ }^{11}$ From Ref. 12, the leading edge (perpendicular to target) expansion velocity of $\approx 1.4 \mathrm{~cm} / \mu \mathrm{s}$ in vacuum has been measured which corresponds to an initial (perpendicular to target) plume temperature of $3.6 \mathrm{eV}$. It is important to clarify that this perpendicular expansion velocity is higher than the parallel velocity, which has been inferred from previous experimental measurements (Ref. 12, Figs. $9 \mathrm{i}, \mathrm{j}$ ) to be about $0.18 \mathrm{~cm} / \mu \mathrm{s}$.

From Ref. 11, the electron density profile of the ablation plume has been measured at various times. Typically, at 50 $\mathrm{ns}$, the measured electron density is about $3 \times 10^{17} \mathrm{~cm}^{-3}$ at $0.3 \mathrm{~mm}$ above the target surface so this density can approach $10^{18}-10^{19} \mathrm{~cm}^{-3}$ at the surface. Thus, in this paper, we consider a rather dense plasma (residual plume) moving up to 1 $\mathrm{cm} / \mu$ s parallel to the target surface, which produces the wavelike pattern. Contrary to the roughness at the edge, we have a rather smooth surface with a regular ripple pattern in the order $30 \mu \mathrm{m}$ spacing in the center of the laser spot.

\section{THE MODEL}

In a sharp boundary model (Fig. 3), the molten aluminum is located in region $\mathrm{I}(z<0)$ and the plasma plume is located in region II $(z>0)$; the interface at $z=0$. In the description of Kelvin-Helmholtz instability, we consider a general density profile $\rho(z)$, and general velocity profile $U(z)$. We may also include the effects of surface tension, viscosity, and gravity. By combining the equation of motion, continuity equation, incompressible condition and perturbation of the form $\exp \left(i k_{x} x+i k_{y} y-i \omega t\right)$, where $\omega=\left(\omega_{r}+i \gamma\right)$, we obtain the governing equation ${ }^{22}$ for the perturbed velocity, $u$ :

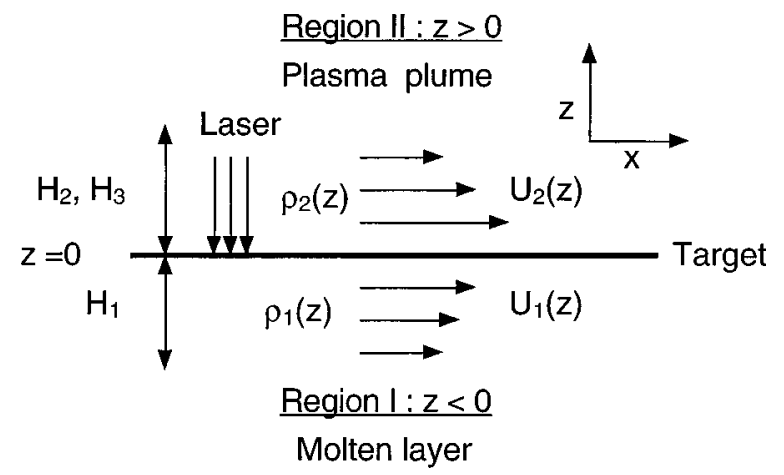

FIG. 3. The model of 2 layers of fluids in relative motion in $x$-direction. $\rho(z)$ and $U(z)$ are the equilibrium mass density and horizontal velocity, where subscript ' 1 ' is for the molten layer $(z<0)$ and ' 2 ' is for the plasma plume $(z>0)$.

$$
\begin{aligned}
& \left(k_{x} U-\omega\right)\left(\frac{d^{2}}{d z^{2}}-k^{2}\right) u-k_{x} u \frac{d^{2} U}{d z^{2}}+\frac{1}{\rho} \frac{d \rho}{d z} \\
& \quad \times\left(\left(k_{x} U-\omega\right) \frac{d u}{d z}-k_{x} u \frac{d U}{d z}-u \frac{g k^{2}}{k_{x} U-\omega}\right) \\
& +u \frac{k^{4} \sigma}{\rho\left(k_{x} U-\omega\right)} \delta(z)+i \nu\left(\frac{d^{4}}{d z^{4}}-2 k^{2} \frac{d^{2}}{d z^{2}}+k^{4}\right) u=0 .
\end{aligned}
$$

Here, $k^{2}=k_{x}^{2}+k_{y}^{2}, g$ is the gravity acceleration, $\sigma$ is surface tension constant, $\nu$ is kinematic viscosity constant, and $\delta(z)$ is the Dirac delta function. To simplify Eq. (1), we compare the magnitude of various terms: gravity, surface tension, and viscosity. From the available experimental data, we notice that $\lambda \equiv 2 \pi / k$ is on the order of $30 \mu \mathrm{m}$. We then find that surface tension is the dominant term, with $\sigma k^{3} / \rho_{1} \gg\left(\nu k^{2}\right)^{2}$ $\gg g k$ (Ref. 23).

In region $\mathrm{I}(z<0)$, we assume that the molten layer has a thickness of $H_{1}$, with a uniform density $\rho_{1}$ and uniform velocity $U_{1}$ (which may be taken to be zero). Equation (1) reduces to

$$
\left(k_{x} U_{1}-\omega\right)\left(\frac{d^{2}}{d z^{2}}-k^{2}\right) u_{1}=0 .
$$

In region II $(z>0)$, we assume that the high-pressure plasma plume gives rise to a wind, with density $\rho_{2} \equiv \rho_{2}(z)$ and velocity, $U_{2} \equiv U_{2}(z)$. We assume that the phase velocity of the surface wave, $\omega / k_{x}$ is much less than the equilibrium velocity, $U_{2}\left(U_{2} \gg \omega / k_{x}\right.$, to be verified later). Equation (1) becomes

$$
\begin{gathered}
\left(k_{x} U_{2}-\omega\right)\left[\frac{d^{2}}{d z^{2}}+\frac{1}{\rho_{2}} \frac{d \rho_{2}}{d z} \frac{d}{d z}-\left(k^{2}+\frac{1}{\rho_{2}} \frac{d \rho_{2}}{d z} \frac{1}{U_{2}} \frac{d U_{2}}{d z}\right.\right. \\
\left.\left.+\frac{1}{U_{2}} \frac{d^{2} U_{2}}{d z^{2}}\right)\right] u_{2}=0 .
\end{gathered}
$$

In order to calculate the growth rate of the instability, we solve Eqs. (2) and (3) in the region $z<0$ and $z>0$ for $u_{1}$ and $u_{2}$, respectively, and integrate Eq. (1) at the boundary from $z=0^{-}$to $0^{+}$. To simplify the calculation, let $\rho_{2}(z)$ $=\rho_{2 o} \exp \left(-z / H_{2}\right)$ and $U_{2}(z)=U_{2 o} \exp \left(-z / H_{3}\right)$, where $H_{2}$ 
is the density scale length of the plume and $\mathrm{H}_{3}$ is the velocity shear scale length of the plume. It is clear that $U_{2 o} \gg U_{1}$ and $\rho_{1} \gg \rho_{2 o}$. We then obtain the growth rate

$$
\gamma=\sqrt{\tanh \left(\Lambda_{1}\right)\left[\left(\Phi \rho_{2 o} U_{2 o}^{2} k^{2}-k^{3} \sigma\right) / \rho_{1}\right]},
$$

and the real part of the frequency

$$
\omega_{r}=k U_{2 o}\left[\frac{\rho_{2 o}}{\rho_{1}} \tanh \left(\Lambda_{1}\right) \Phi+\frac{U_{1}}{U_{2 o}}\right],
$$

where $\Phi \equiv\left[2 \sqrt{\Lambda_{2}^{2}+(r+1 / 2)^{2}}-1\right] / 2 \Lambda_{2} \geqslant 0, \Lambda_{1} \equiv k H_{1}, \Lambda_{2}$ $\equiv k H_{2}$, and $r \equiv H_{2} / H_{3}$ are normalized parameters. In obtaining Eqs. (4) and (5), the boundary conditions $u_{1}\left(-H_{1}\right)=0$, $u_{2}(\infty)=0$ and $u_{2}(0)-u_{1}(0)=U_{2}(0)-U_{1}(0)$, as well as the assumption $k_{x} \approx k$ have been used. Note that Eq. (4) can be extended to include the Rayleigh-Taylor instability and gravitation effects, by adding the term $\rho_{1} k(\alpha-g)$ in the numerator in the square bracket of Eq. (4), where $\alpha$ is the acceleration force which accelerates vertically from the molten layer to the plasma plume layer, and $g$ is gravitational acceleration. In this article, we estimate that these effects are negligible due to the high plasma plume velocity. ${ }^{10-16}$

Since there are many parameters that are not readily available from experiments (such as $H_{1}, H_{2}$ and $H_{3}$ ), to proceed further, we postulate that the wavelike pattern that is observed in experiments (Fig. 1) corresponds to the wavenumber that yields the maximum growth. Then by varying $H_{1}, H_{2}$, and $H_{3}$, we may infer what would be the temporal growth rate of the Kelvin-Helmholtz instability, and the plasma wind kinetic energy density $\Delta \equiv \rho_{2 o} U_{2 o}^{2}$ that is required so as to produce such a growth with the observed wave number. Thus, by setting $d \gamma / d k=0$, we obtain the equation that maximizes the growth rate and the energy density that is required to yield such a growth with the observed wave number $k$. The normalized growth rate, $\bar{\gamma}$, and the corresponding normalized plasma plume's energy density, $\bar{\Delta}$, are given by

$$
\bar{\gamma} \equiv \frac{\gamma}{\gamma_{\infty}}=\sqrt{\frac{2 \tanh \Lambda_{1}\left(\Phi-\frac{\Lambda_{2}}{\left(2 \Lambda_{2} \Phi+1\right)}\right)}{\Phi\left(\frac{1}{2}+\frac{\Lambda_{1}}{\sinh 2 \Lambda_{1}}\right)+\frac{\Lambda_{2}}{2 \Lambda_{2} \Phi+1}}},
$$

and

$$
\bar{\Delta} \equiv \frac{\Delta}{\Delta_{\infty}}=\left[\frac{1+\frac{2 \Lambda_{1}}{3 \sinh 2 \Lambda_{1}}}{\Phi\left(\frac{1}{2}+\frac{\Lambda_{1}}{\sinh 2 \Lambda_{1}}\right)+\frac{\Lambda_{2}}{2 \Lambda_{2} \Phi+1}}\right] .
$$

Here $\left[\gamma_{\infty}, \Delta_{\infty}\right]=\left[\left(\sigma k^{3} / 2 \rho_{1}\right)^{1 / 2}, 3 \sigma k / 2\right]$, the quantities corresponding to $H_{1}, H_{2}, H_{3} \rightarrow \infty$. For $\lambda=30 \mu \mathrm{m}$, we have $\gamma_{\infty}$ $\approx 1.3 \mu \mathrm{s}^{-1}$, and $\Delta_{\infty} \approx 0.272 \mathrm{~J} / \mathrm{cm}^{3}$, using $\rho=2.37 \mathrm{~g} / \mathrm{cm}^{3}$, $\sigma=0.865 \mathrm{~N} / \mathrm{m}, \quad \nu=1.055 \times 10^{-2} \mathrm{~cm}^{2} / \mathrm{s}$ and $g=9.8 \mathrm{~m} / \mathrm{s}^{2}$ (from Ref. 23).

Traditionally, Eq. (7) gives the most probable period $\lambda$ of the surface wave when $\Delta, H_{1}, H_{2}, H_{3}$ and the thermophysical properties are given, and the growth rate at this $\lambda$ is calculated from Eq. (6). Here, we keep $H_{1}, H_{2}$, and $H_{3}$ as free parameters, and use the experimental value of $\lambda$ to esti-

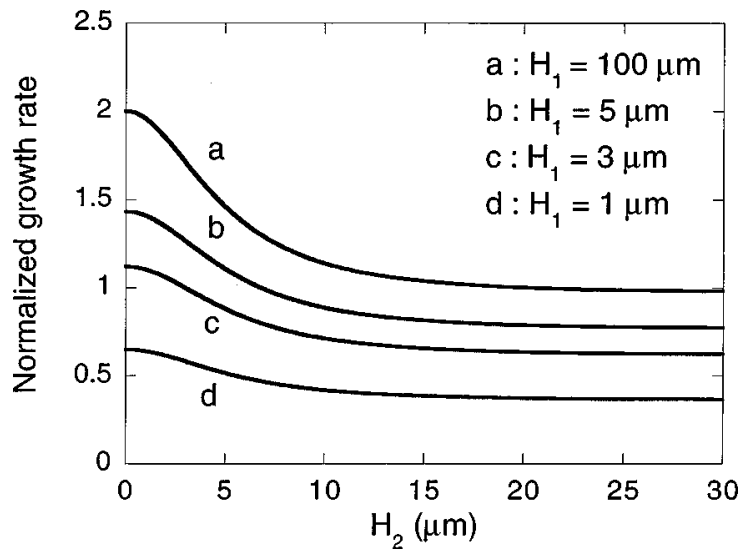

FIG. 4. Normalized growth rate, $\bar{\gamma} \equiv \gamma / \gamma_{\infty}$ as a function of $H_{2}$ for various $H_{1}$ at $\lambda=30 \mu \mathrm{m}$ and $r=1\left(r \equiv H_{2} / H_{3}\right) . H_{2}, H_{3}$, and $H_{1}$ are, respectively, the characteristic scale length of the plume's density profile, the plume's velocity profile and molten layer of $\mathrm{Al}$.

mate the growth rate with Eq. (6) and calculate the corresponding plasma energy density as a function of $\mathrm{H}_{1}, \mathrm{H}_{2}$, $\mathrm{H}_{3}$.

By setting $\lambda=30 \mu \mathrm{m}$, we show the dependence of the growth rate on $H_{1}$ and $H_{2}$. Figure 4 shows that the growth rate increases with $H_{1}$ and decreases with $H_{2}$ for $r$ $\equiv H_{2} / H_{3}=1$ (spatial variation of the plume density profile is assumed to be equal to that of parallel velocity profile). The growth rate can be twice $\gamma_{\infty}$ at high $H_{1}$ and low $H_{2}$, and diminishes to zero at low $H_{1}$. We also calculate the corresponding energy density needed to drive the surface instability at $\lambda=30 \mu \mathrm{m}$. The dependence of $\bar{\Delta}$ on $H_{1}$ and $H_{2}$ are given in Fig. 5; it increases with $H_{1}$ and $H_{2}$. However, for a wide range of $H_{1}(1-100 \mu \mathrm{m}), \bar{\Delta}$ does not change much, with an asymptotic value between 0.9 and 1.1 for $\mathrm{H}_{2}$ $>5 \mu \mathrm{m}$.

In order to calculate the growth rate of these large surface structures and energy density of the ablation plume, we have to estimate $H_{1}, H_{2}$, and $H_{3}$. In the fluence range of a few $\mathrm{J} / \mathrm{cm}^{2}$ of laser ablation, $H_{1}$ is typically a few microns. By setting $\lambda=30 \mu \mathrm{m}, H_{1}=2 \mu \mathrm{m}$, and $r=1$, the normalized

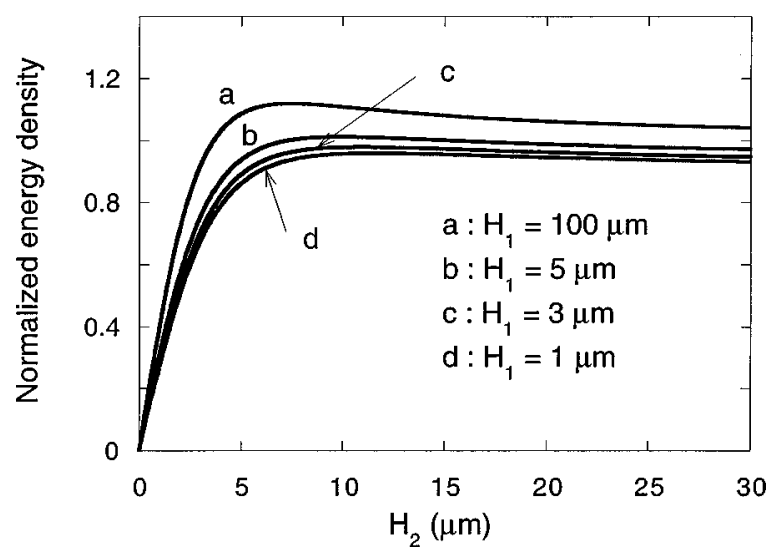

FIG. 5. Normalized energy density, $\bar{\Delta} \equiv \Delta / \Delta_{\infty}$ as a function of $H_{2}$ for various $H_{1}$ at $\lambda=30 \mu \mathrm{m}$ and $r=1\left(r \equiv H_{2} / H_{3}\right) . H_{2}, H_{3}$, and $H_{1}$ are, respectively, the characteristic scale length of the plume's density profile, the plume's velocity profile and molten layer of Al. 


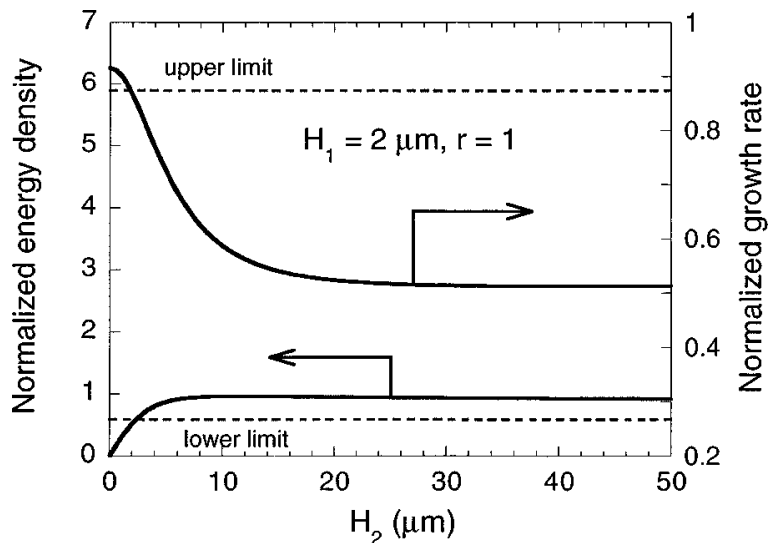

FIG. 6. Normalized growth rate, $\bar{\gamma} \equiv \gamma / \gamma_{\infty}$ and normalized energy density, $\bar{\Delta} \equiv \Delta / \Delta_{\infty}$ as a function of $H_{2}$ at $\lambda=30 \mu \mathrm{m}, H_{1}=2 \mu \mathrm{m}$, and $r=1(r$ $\left.\equiv H_{2} / H_{3}\right)$. The dashed lines are the upper and lower limits of the measured $\bar{\Delta}\left(\propto n_{e} T_{e}\right)$, where $n_{e} \approx 10^{19} \mathrm{~cm}^{-3}$ and $T_{e} \approx 1 \mathrm{eV}$ (upper limit) and $0.1 \mathrm{eV}$ (lower limit).

energy density and growth rate are given in Fig. 6. To ensure that $\mathrm{KH}$ instability is indeed a possible mechanism for the growth at $\lambda=30 \mu \mathrm{m}$, we compare the calculated $\bar{\Delta}$ with measured $\bar{\Delta}$, by setting $\Delta \approx n_{e} T_{e}$. Here, $n_{e}$ is the electron density at the target surface $\left(\approx 10^{19} \mathrm{~cm}^{-3}\right)$ and $T_{e}$ is electron temperature corresponding to the parallel velocity ${ }^{14}(1 \mathrm{eV}$ for upper limit and $0.1 \mathrm{eV}$ for lower limit), where both $n_{e}$ and $T_{e}$ were previously measured from this experiment. ${ }^{11-12,24} \mathrm{In}$ Fig. 6 , the upper and lower limit of the measured $\bar{\Delta}$ are shown. As can be seen, the calculated $\bar{\Delta}$ lies within the experimental range, and the calculated growth rate is about $0.66 \mu \mathrm{s}^{-1}$ at $H_{2}>20 \mu \mathrm{m}$.

\section{CUMULATIVE GROWTH}

The above parametric study of the temporal growth, as a function of $H_{1}, H_{2}$, and $H_{3}$, allows us to assess the total growth after $N$ laser pulses, each pulse lasting $40 \mathrm{~ns}$. We wish to calculate the cumulative growth rate, $\gamma^{*}\left(=\Sigma_{i}^{N} \gamma_{i}\right)$ after $N$ laser pulses of irradiation. As can be seen in Figs. 1 and 2, molten surface waves of material that have been solidified have a rather smooth ripple with period of about 30 $\mu \mathrm{m}$ in the center of the laser spot. However, at the edge of the crater, we found material piled up in conical structures with height 50 to $100 \mu \mathrm{m}$ (see SEM image in Ref. 2). Thus, we assume that $H_{2}(N)=H_{3}(N)$ is a linearly increasing function with $N$, up to $100 \mu \mathrm{m}$ at $N=250$ and $H_{1}(N)$ is a constant $(=1,2,3 \mu \mathrm{m}){ }^{25}$ The increase in the scales $H_{2}$ and $H_{3}$ in the plasma plume, as $N$ increases, is on account of the observed buildup of the surface roughness as $N$ increases. The spatial extent of the plasma, as measured by $\mathrm{H}_{2}$ and $\mathrm{H}_{3}$, is then expected to increase with $N$. It turns out that the final result is not sensitive to the precise values of $\mathrm{H}_{2}$ and $\mathrm{H}_{3}$, with $H_{2}, H_{3}>10 \mu \mathrm{m}$, but it is more sensitive to the magnitude of $H_{1}$ (see Fig. 4).

Using such a model, we show the growth of $\exp \left(\gamma^{*} \tau\right)$ in Fig. 7, up to $N=250$, where $\tau$ is set to be the laser pulselength of $40 \mathrm{~ns}$. The effective growth for 250 pulses is about

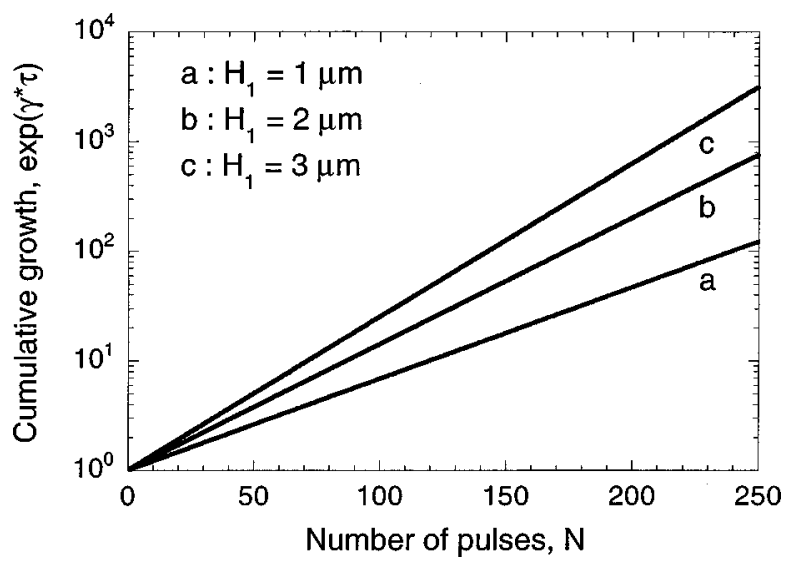

FIG. 7. Calculated cumulative growth, as a function of $N$ (number of pulses) of $\mathrm{KrF}$ excimer laser ablation $\left(248 \mathrm{~nm}, 40 \mathrm{~ns}, 5 \mathrm{~J} / \mathrm{cm}^{2}\right)$ on a pure aluminum target for $H_{1}=1,2,3 \mu \mathrm{m}$.

3000 times for $H_{1}=3 \mu \mathrm{m}$, which may be deemed reasonable, considering all the approximations involved. The issue of saturation remains to be studied.

In ablation experiments, the plume's density profile is time dependent, which, roughly speaking, evolves from $\exp (-z / H)^{2}$ and to a profile whose peak density is located far above the interface surface, ${ }^{26}$ after a much longer time scale than the laser pulse length (due to plume expansion from the interface surface). We have assumed a constant profile within the laser pulselength time scale in this model, but due to the lower gradient $(d \rho / d z)$ at the interface surface of these evolved density profiles at later time, the contributions of this expanding plume to growth become negligible.

To verify our assumption of $U_{2} \gg \omega / k_{x}$, under the conditions $U_{2 o} \gg U_{1}$ and $\rho_{1} \gg \rho_{2 o}$, we found that $\omega_{r} \ll k U_{2 o}$ [see Eq. (5)], and the maximum of $\gamma / k$ (and $\omega_{r} / k$ ) is about 1240 $\mathrm{cm} / \mathrm{s}$. Thus the phase velocity of the surface wave is indeed much slower than any velocity in the plasma plume, which is in the range of $10^{5}-10^{6} \mathrm{~cm} / \mathrm{s} .{ }^{11,12}$

\section{ACKNOWLEDGMENTS}

This research was supported by National Science Foundation Grant No. CTS-9522282. H.L.S. and J.S.L. acknowledge previous support from National Science Foundation Graduate Fellowships. The authors also acknowledge the support of Sandia National Laboratory. They wish to thank the referee for many helpful comments.

${ }^{1}$ D. B. Chrisey, and G. K. Hubler, Pulsed Laser Deposition of Thin films (Wiley, New York, 1994).

${ }^{2}$ H. L. Spindler, R. M. Gilgenbach, and J. S. Lash, Appl. Phys. Lett. 68, 3245 (1996)

${ }^{3}$ S. R. Foltyn, R. C. Dye, K. C. Ott, E. Peterson, K. M. Hubbard, W. Hutchinson, R. E. Muenchausen, R. C. Estler, and X. D. Wu, Appl. Phys. Lett. 59, 594 (1991).

${ }^{4}$ R. Kelly and J. E. Rothenberg, Nucl. Instrum. Methods Phys. Res. B 7/8, 755 (1985); R. Kelly, J. J. Cuomo, P. A. Leary, J. E. Rothenberg, B. E. Braren, and C. F. Aliotta, ibid. 9, 329 (1985); J. E. Rothenberg and R. Kelly, ibid. 1, 291 (1984).

${ }^{5}$ H. M. van Driel, J. E. Sipe, and J. F. Young, Phys. Rev. Lett. 49, 1955 (1982); J. E. Sipe, J. F. Young, J. S. Preston, and H. M. van Driel, Phys. Rev. B 27, 1141 (1983). 
${ }^{6}$ F. Keilmann, Phys. Rev. Lett. 51, 2097 (1983); S. R. J. Brueck, and D. J. Ehrlich, ibid. 48, 1678 (1982).

${ }^{7}$ S. A. Akhmanov, V. I. Emel'yanov, N. I. Koroteev, and V. N. Seminogov, Sov. Phys. Usp. 28, 1084 (1985); O. Bostanjoglo and T. Nick, J. Appl. Phys. 79, 8725 (1996).

${ }^{8}$ A. B. Brailovsky, S. V. Gaponov, and V. I. Luchin, Appl. Phys. A: Solids Surf. 61, 81 (1995).

${ }^{9}$ L. K. Ang, Y. Y. Lau, R. M. Gilgenbach, and H. L. Spindler, Appl. Phys. Lett. 70, 696 (1997).

${ }^{10}$ J. S. Lash, Ph.D. dissertation, University of Michigan, 1996.

${ }^{11}$ R. M. Gilgenbach, C. H. Ching, J. S. Lash, and R. A. Lindley, Phys. Plasmas 1, 1619 (1994).

${ }^{12}$ R. A. Lindley, R. M. Gilgenbach, C. H. Ching, and J. S. Lash, J. Appl. Phys. 76, 5457 (1994).

${ }^{13}$ R. M. Gilgenbach and P. L. G. Ventzek, Appl. Phys. Lett. 58, 1597 (1991).

${ }^{14}$ P. L. G. Ventzek, R. M. Gilgenbach, C. H. Ching, and R. A. Lindley, J. Appl. Phys. 72, 1696 (1992).

${ }^{15}$ P. L. G. Ventzek, R. M. Gilgenbach, J. A. Sell, and D. M. Heffelfinger, J. Appl. Phys. 68, 965 (1990).

${ }^{16}$ P. L. G. Ventzek, R. M. Gilgenbach, D. M. Heffelfinger, and J. A. Sell, J. Appl. Phys. 70, 587 (1991).

${ }^{17}$ X. Mao and R. E. Russo, Appl. Phys. A: Solids Surf. 64, 1 (1997).

${ }^{18}$ See, e.g., D. Bauerle, in Laser Processing and Chemistry (Springer, Berlin, 1996), p. 409.

${ }^{19}$ RT instability model from Ref. 8 is based on acceleration produced by centrifugal force of molten flow at convex liquid-solid interface, which is a second-order effect (the velocity of molten flow is not high enough to produce a significant force).
${ }^{20}$ T. D. Bennett, C. P. Grigoropoulos, and D. J. Krajnovich, J. Appl. Phys. 77, 849 (1995). Here, we linearly interpolated the acceleration force $(\alpha)$ by using the Eq. (7) in this reference for our experiment of an A1 target ablated by laser intensity of $5 \mathrm{~J} / \mathrm{cm}^{2}$. Note that even without interpolation, using the value from their case of $1 \mathrm{~J} / \mathrm{cm}^{2}$, we have $\lambda_{m}=8 \mu \mathrm{m}$.

${ }^{21}$ S. I. Anisimov and V. A. Khokhlov, Instabilities in Laser-Matter Interaction (Chemical Rubber Co., Boca Raton, 1995).

${ }^{22}$ See, e.g., S. Chandrasekhar, in Hydrodynamic and Hydromagnetic Stability (Dover, New York, 1961), Chap 6. Note that viscosity term has been included.

${ }^{23}$ The difference in the inequality is of order $10^{3}$, for $\rho=$ density of molten $\mathrm{Al}=2.37 \mathrm{~g} / \mathrm{cm}^{3}, \quad \sigma=0.865 \quad \mathrm{~N} / \mathrm{m}, \quad \nu=1.055 \times 10^{-2} \mathrm{~cm}^{2} / \mathrm{s}, \quad$ and $g$ $=9.8 \mathrm{~m} / \mathrm{s}^{2}$.

${ }^{24}$ In the ablation plume, the ion component can been ignored as ion temperature is much lower than electron temperature. Note that the electron density is difficult to measure at the target surface, here we have estimated it is about $10^{19} \mathrm{~cm}^{-3}$ (two orders higher than what we measured at $0.3 \mathrm{~mm}$ above the target surface).

${ }^{25}$ The depth of the molten aluminum, $H_{1}$, is likewise expected to increase with $N$ for small $N$. However, for larger values of $N$, the plasma plume becomes substantial and it may begin to absorb (and reflect) the laser light, leaving a much reduced fraction of laser energy that could reach the aluminum surface (Ref. 17). Thus, $H_{1}$ is expected to be reduced as $N$ becomes large. However, for simplicity we assume that $H_{1}$ does not change much, which is around $1-3 \mu \mathrm{m}$.

${ }^{26}$ A. Mele, A. G. Guidoni, R. Kelly, A. Miotello, S. Orlando, R. Teghil, and C. Flamini, Nucl. Instrum. Methods Phys. Res. B 116, 257 (1996). 